ISSN 2693-2504

\title{
Analytical Cloud system ERI - a Novel Approach in Energy Medicine
}

Journal of Bioscience \& Biomedical Engineering

Brief Report

Dr. Igor Orzhelskyi, Dr. Andrey Kuznetsov, MSc. Elena van Dijk*

*Correspondence author

CME SWISS, Complete Medical Expert in Digital Health

\author{
Elena van Dijk \\ CME SWISS \\ Complete Medical Expert in Digital Health
}

Submitted : 19 Mar 2021 ; Published : 5 Apr 2021

\section{Introduction}

It is well acknowledged that health is a multidimensional concept and is shaped by biological, physical, psychological, social, economic, cultural and political factors.

Access to basic needs like food, safe water supply, housing, sanitation and health services

influences the health status of a population. Other important factor influencing the quality of life is often overlooked. It is the individuality and unique needs of each person to maintain a

healthy, productive living. The evaluation of this subject need's plurality of strategies, which should be a part of continuous and comprehensive plan for healthier life style.

CME SWISS AG has developed a tool, the "Analytical Cloud System ERI" to measure a human biofield, digitize it and use AI for a deep analysis and detection of pathology. The device provides a unique approach to improve and maintain health and quality of life by background electric field analysis and and its correction.

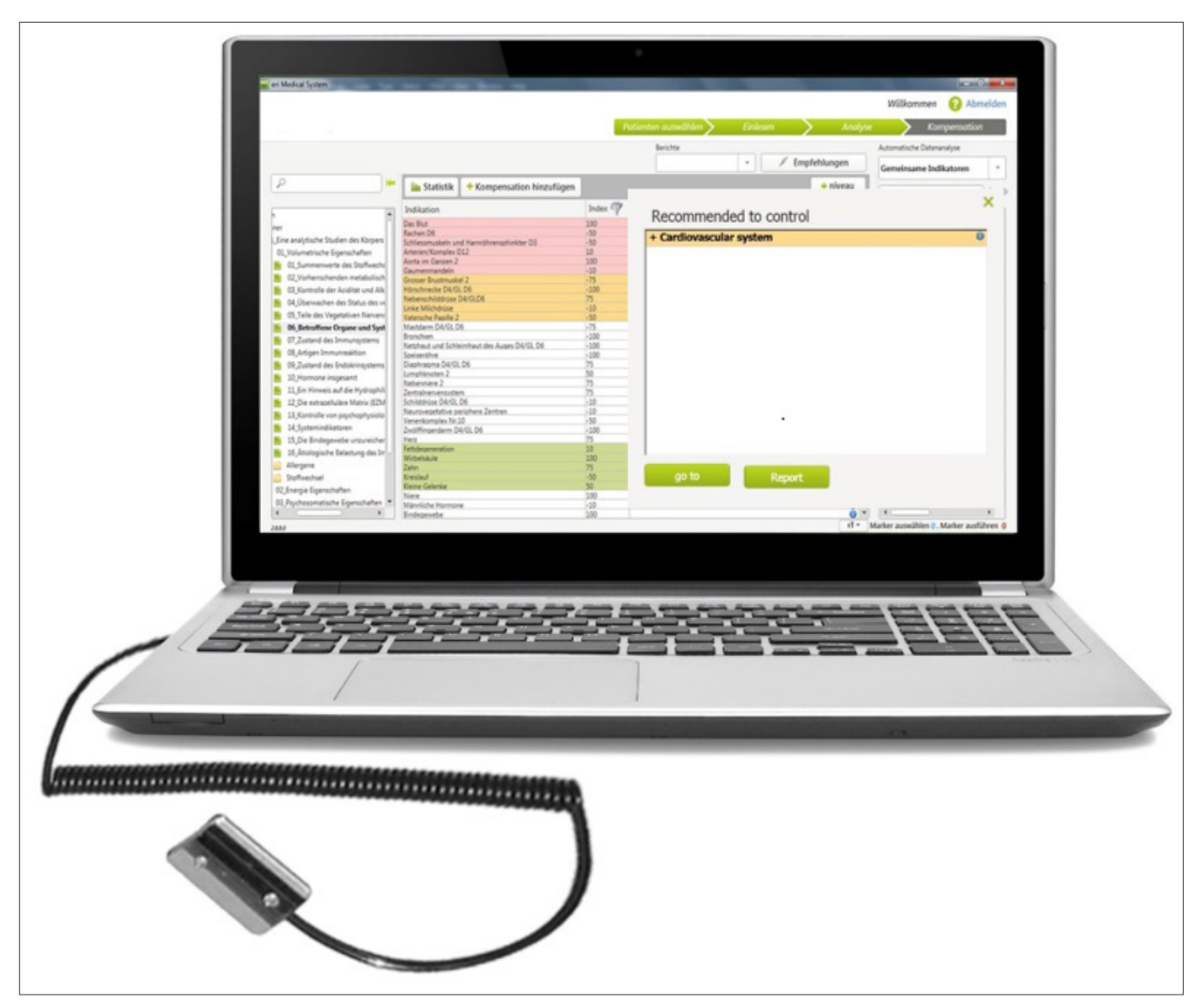


Analyzing background electric fields; observing repeatable patterns in seemingly chaotic processes. Understanding electricity of our own body.

Each matter is made up of atoms, and atoms are made up of protons, neutrons and electrons. Protons have a positive charge, neutrons have a neutral charge, and electrons have a negative charge. When these charges are out of balance, an atom becomes either positively or negatively charged. The switch between one type of charge and the other allows electrons to flow from one atom to another. This flow of electrons, or a negative charge, is what we call electricity. Since our bodies are huge masses of atoms, we can generate electricity.

Electricity is a key to survival.

Low-level energy field of the body

Energy medicine typically involves a low-level energy field interactions.

One possible influence of biofield phenomena is that they may act directly on molecular structures, changing the conformation of molecules in functionally significant ways. Another influence is that they may transfer bioinformation carried by exceedingly small energy signals interacting directly with the energy fields which is more recently known as the biofield.

Moreover, another mystery is that living organisms respond to extremely low-level nonionizing electromagnetic fields, displaying a variety of effects ranging from cellular and subcellular scales to the level of brain, emotions, and behavior. These fields may be beneficial (therapeutic), deleterious (electromagnetic pollution), or neutral. Then, the mystery of embryonic development from the fertilized egg to an organized integral unit should be considered, which may also involve innate energy fields, starting with the initial polarization of the fertilized egg.

Although these phenomena involve an integral and dynamic wholeness that challenge the power of molecular explanation, another biophysical view of life has been offered that may help explain them. Living systems may be regarded as complex, nonlinear, dynamic, self-organizing systems of energetic and field phenomena. At the highest level of organization, each life form may possess an innate biologic field, or biofield, a complex, dynamic, weak energy field involved in maintaining the integrity of the whole organism, regulating its physiologic and biochemical responses, and integral to development, healing, and regeneration.

One of the leading biometrics fields that characterize the human condition and the processes taking place in the body, is an electric field.

Electric field, as one of the components of the electromagnetic field, gives the most complete display of the actual dynamic of processes and their change over time. This is due to the fact that all the changes in the dynamics of the electric field take place almost instantaneously.

- Development of the physic-mathematical medicine.

- Healthcare systems/devices based on the energy/electrical readings of the body

- Magnetic and Electric Fields in Life
Unlike the electric field, the magnetic field is not fundamental, that is, the concept of a magnetic force is not a separate force, but arises directly from the electric force.

This is an important factor for understand, why in the ERI system principle of dynamic analysis of the background electric field (not magnetic) is implemented.

Electrical currents, along with their associated magnetic fields, can be found in the body (Becker and Selden, 1985). The electrical and magnetic fields of the human body are complex and dynamic and are associated with dynamical processes such as heart and brain function, blood and lymph flow, ion transport across cell membranes, and many other biologic processes on many different scales. These phenomena all contribute various field components to the biofield.

In addition, a broad spectrum of radiant energies exists known as electromagnetic waves, ranging from the ultra-low, extremely low, very-low, low, and medium broadcast waves; extremely high - frequency broadcast waves; microwaves; infrared rays; visible light rays; and even ultraviolet radiation, all emanating from the human body. The peak intensity of the electromagnetic radiation of the human biofield is in the infrared region of the electromagnetic spectrum, in the range of 4 to 20 microns in wavelength. The belief is that much of this emission, particularly in the infrared region, is from thermal effects associated with metabolism.

\section{Human Biofield}

The fields of the human body may also be influenced by the fields of nearby organisms, the biosphere, and even the earth and cosmos, especially geocosmic rhythms.

The human biofield may carry novel information of diagnostic and predictive value for medicine.

The "Analytical Cloud System ERI" can record the human biofield, its dynamic tendencies and complexities.

By analyzing various aspects of the biofield, ERI system is able to recognize organ and tissue dysfunctions and subsequently correct them. The systems may also be able to use biofield analysis to predict whether the effect of a particular course of therapy will be effective or ineffective, depending on whether it improves or thwarts the biofield.

Historical reference of origins of spectral analysis, which is the part of the science behind the ERI system. Fraunhofer Lines 

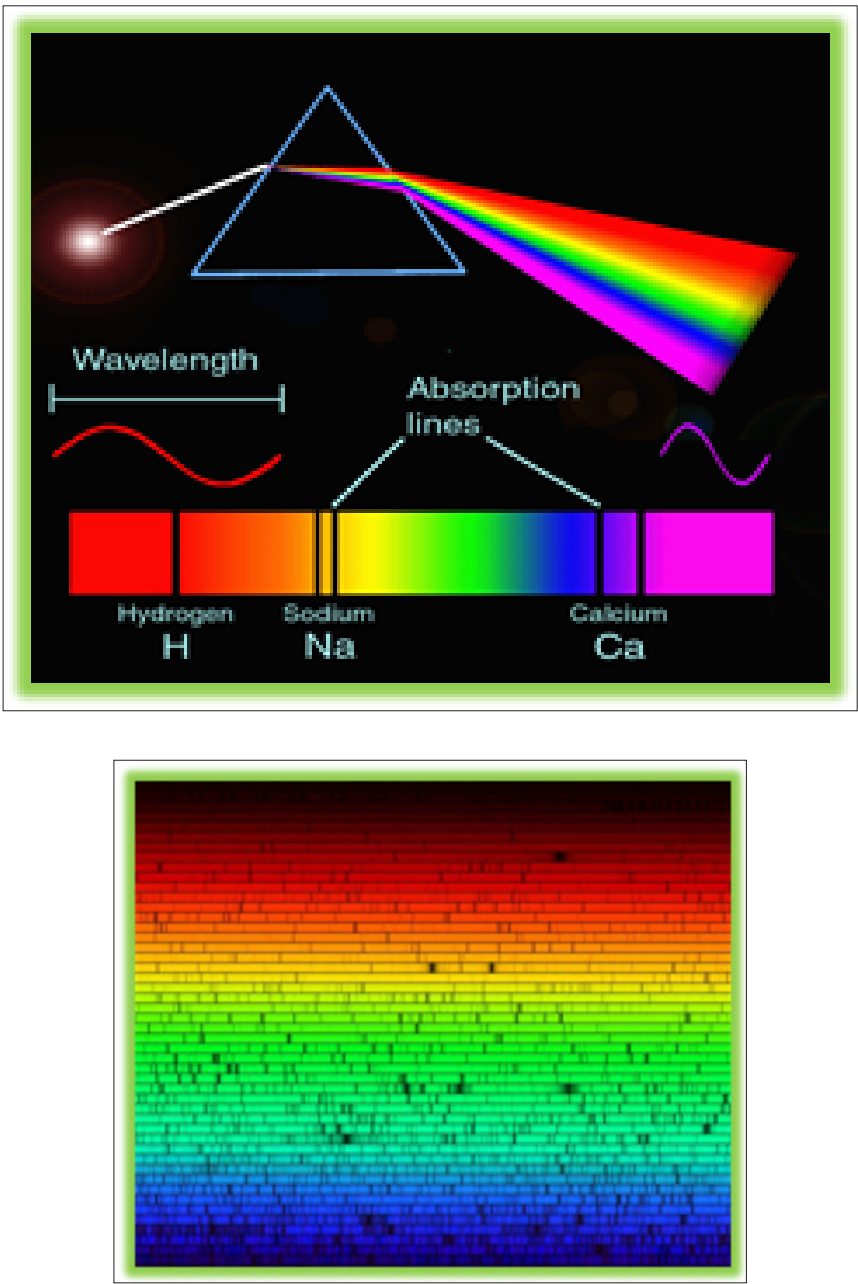

In physics and optics, the Fraunhofer lines are a set of spectral lines named for the German physicist Joseph von Fraunhofer (1787-1826). The lines were originally observed as dark features in the optical spectrum of the Sun.

The English chemist William Hyde Wollaston was in 1802 the first person to note the appearance of a number of dark features in the solar spectrum. In 1814, Fraunhofer independently rediscovered the lines and began a systematic study and careful measurement of the wavelength of these features. In all, he mapped over 570 lines, and designated the principal features with the letters A through $\mathrm{K}$, and weaker lines with other letters.

It was later discovered by inventors of spectroscope Georg Kirchoff and Robert Wilhelm Bunsen that each chemical element was associated with a set of spectral lines.

\section{ERI systems as a type of spectra analyzer}

Emission spectroscopy uses the range of micro-electric waves radiated by object's such as molecules, atoms, and subatomic particles. Micro-electric emission can be from a variety of sources, including chemical reactions. Emission spectroscopy analyzes certain atomic nuclei to determine different local environments of hydrogen, carbon, or other atoms in the molecule of an organic compound or other compound. This is used to help determine the structure of the compound.

ERI systems analyze certain physical properties by recording the strength and the change of the scattered electric field that an object scatters at certain wavelengths.

Then it transforms an incoming time-domain emission into a spectra sequence.

The electric field of a substance consists of the sum of individual fields of atoms and molecules of the substance whose field is being analyzed. In this way what is being analyzed is the dynamics of the substance.

Visual representation of the electric field of chemical elements

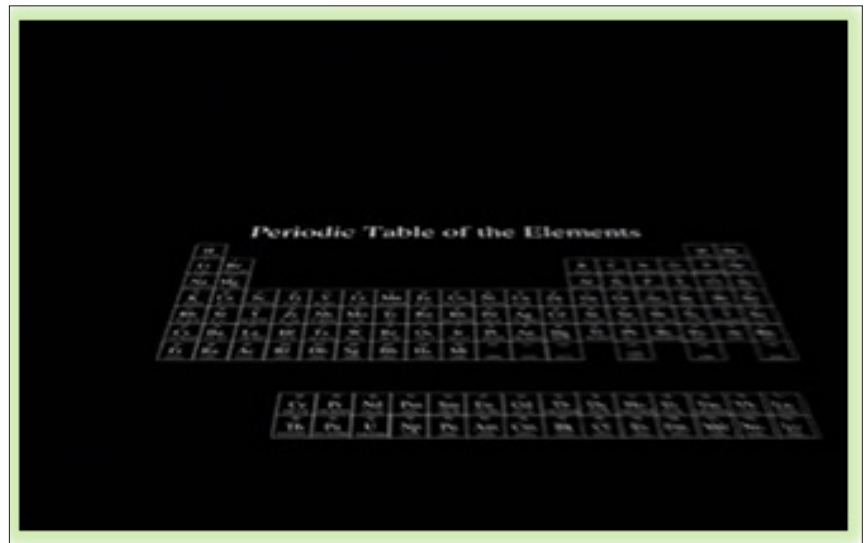

The electric field of a process consists of the electric fields of the substances participating in the process. And, correspondingly, the dynamics of the process is analyzed.

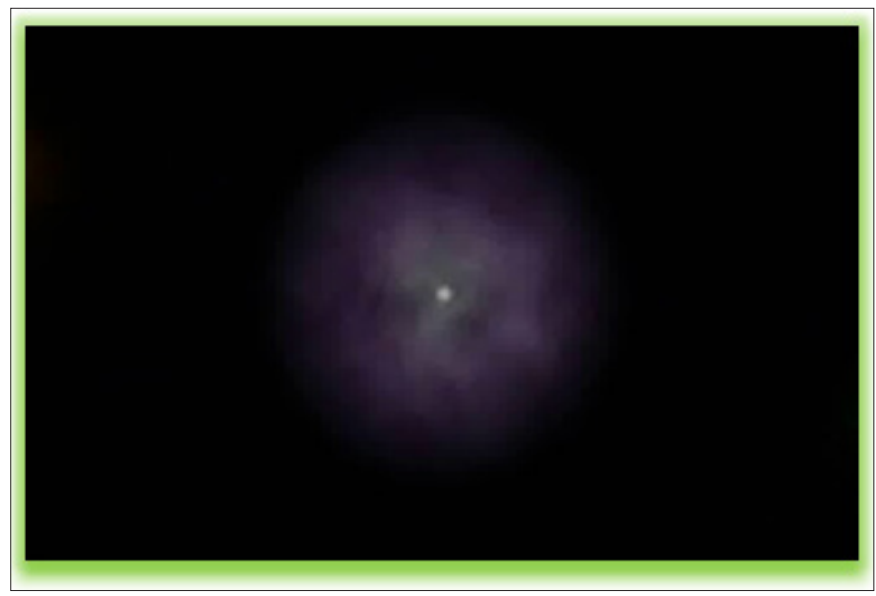

Electric field of a cell recorded by an electronic microscope

Because of the Infinitely large number of substances and processes taking place in the human body as well as in its habitat, the surrounding world, all these processes, in their totality, are of stochastic, noise-like nature. In this way all the processes taking place inside us and in the surrounding world, form the background noise-like electric field.

Direct analysis of the noise-like, stochastic processes is not possible because of the high degree of uncertainty characteris- 
tic of the dynamics of these processes.

That is the reason why the method of digital transformation of the natural, background noise electric field into its digital multidimensional model has been used in the ERI system.
The software application which is an integral part of the ERI system, carries out the spectral dynamic analysis of the obtained digital model, enabling its user to determine the actual health condition of the patient to a high degree of probability.

Visual example of analog-digital transformation of stochastic characteristic into its digital model

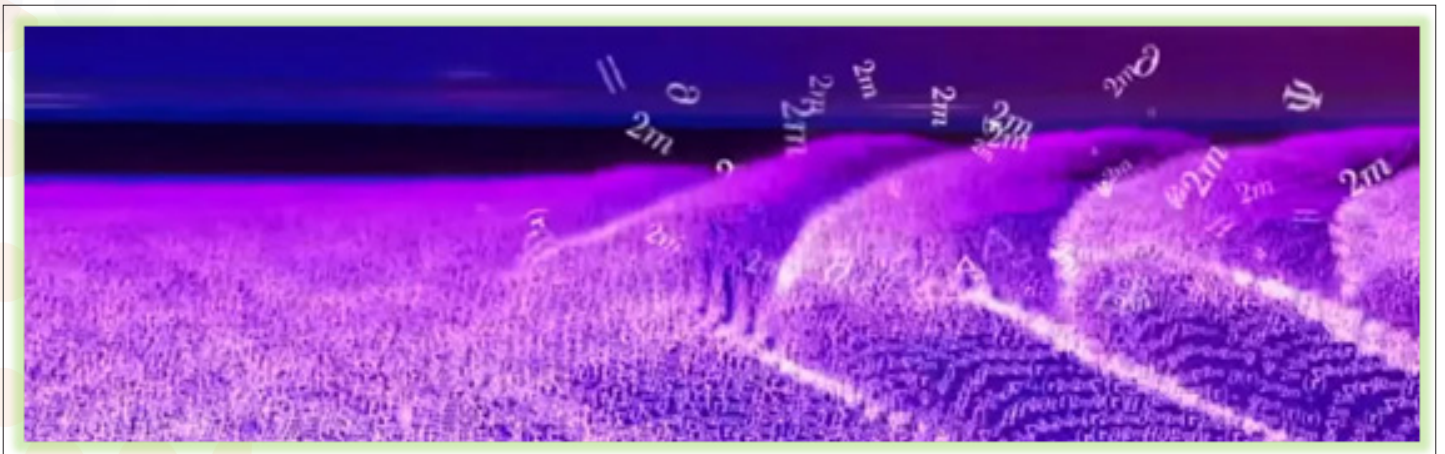

Analog characteristics of the natural electric field are being mathematically transformed to a digital multi-dimensional vector model.

Before recording the patient's characteristics, system operator conducts 3 seconds calibration of the system by recording the characteristic of the ambience of the place of meeting such as practitioner's office. The recorded environmental characteristic parameters will be taken into consideration by the software application when conducting analysis.

While conducting investigation with the help of the ERI system, the sensor - electrode is wrapped in a dry disposable tissue paper and placed on the person's body. The ergonomic shape of the sensor is most convenient for taking it into a hand. While recording the characteristics, the tissue paper ensures the hygiene of the procedure and absence of disturbances in the recorded signal caused by the "galvanic muscle electricity" and the conductivity of the skin.

The time taken for recording the person's characteristics is 40 seconds.

The calculation and filtering of the environmental characteristics takes about $5-10$ seconds.

In this way, by conducting the analysis of the person's characteristic the ERI operator receives the information to evaluate the desired parameters.

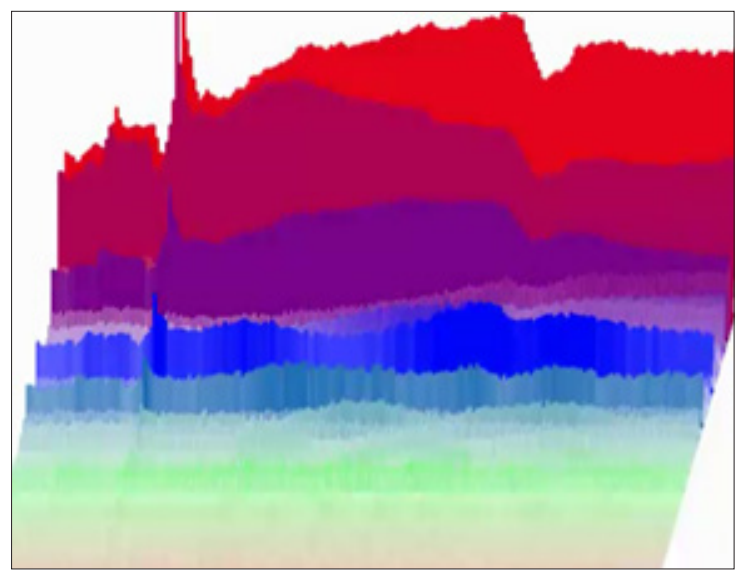

Visual example of analysis of the stochastic field characteristic by the software application.

Visual example of the stochastic characteristic of person's electric field that is recorded by the sensor-electrode of the system

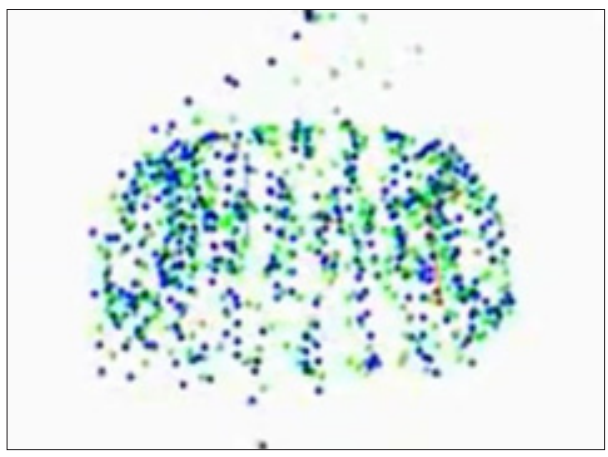

Visual example of obtaining digital coefficients of the model of stochastic characteristic of the electric field of the patient for the subsequent analysis

Spectral dynamic marker consists of several data clusters corresponding to the dynamic processes of any level of complexity. 


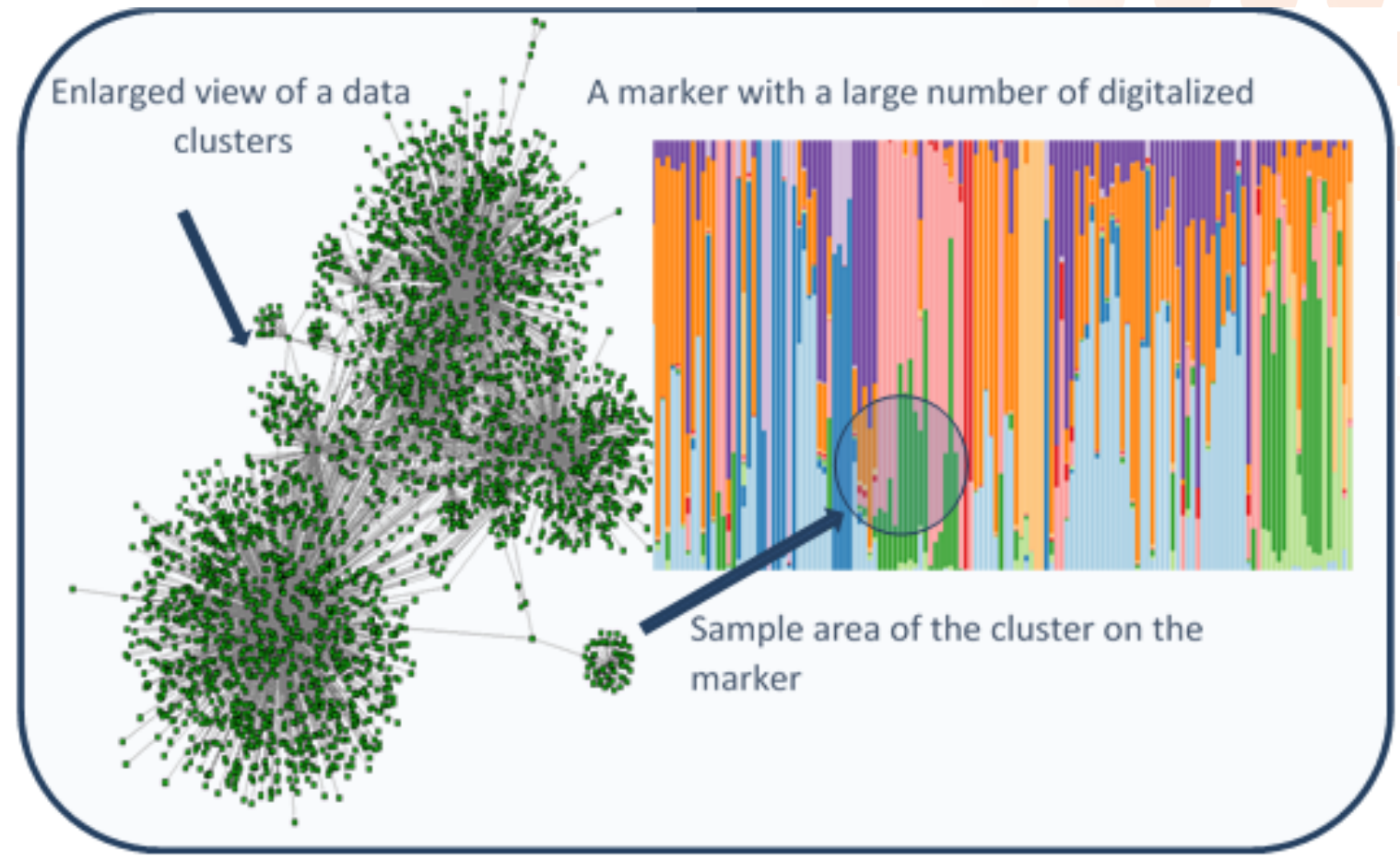

Spectral dynamic markers of: human organ systems; organs themselves and their cells; physiological and negative processes; diseases occurring in human organism; pathogens - fungi, viruses, bacteria, physiological conditions, etc., have been created by the KME specialists in the company's laboratory.

The created markers are then entered into the data base of the system and are used to conduct the analysis by the ERI system operator.

Graphic depiction of the data bases of markers
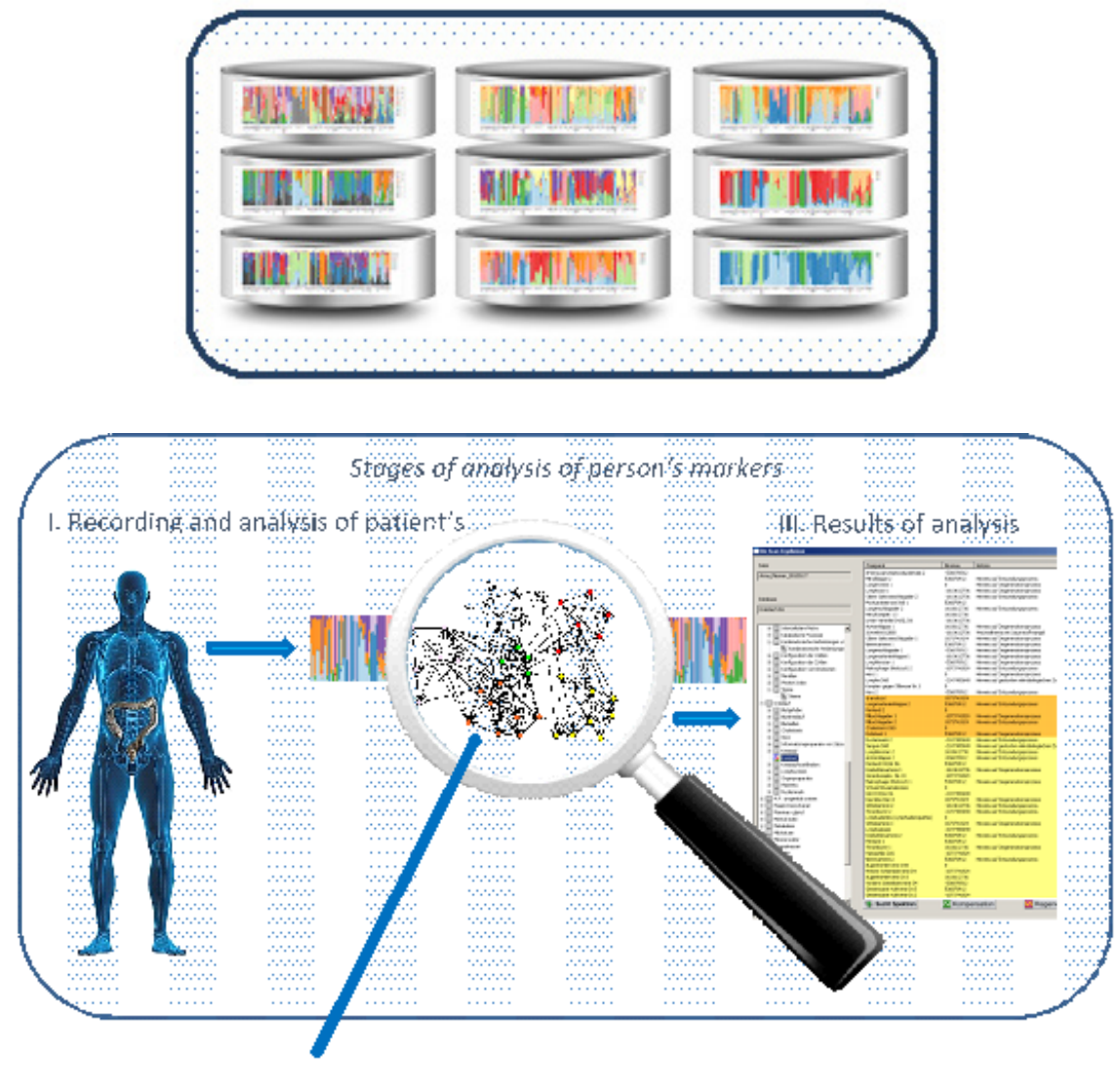
The comparative analysis of the patient's marker with the markers of processes, conditions of organs and their systems and pathogens from the system data base enables the user of the system to make a holistic, comprehensive decision about the health condition.

The results of the analysis are depicted on the screen of the system.

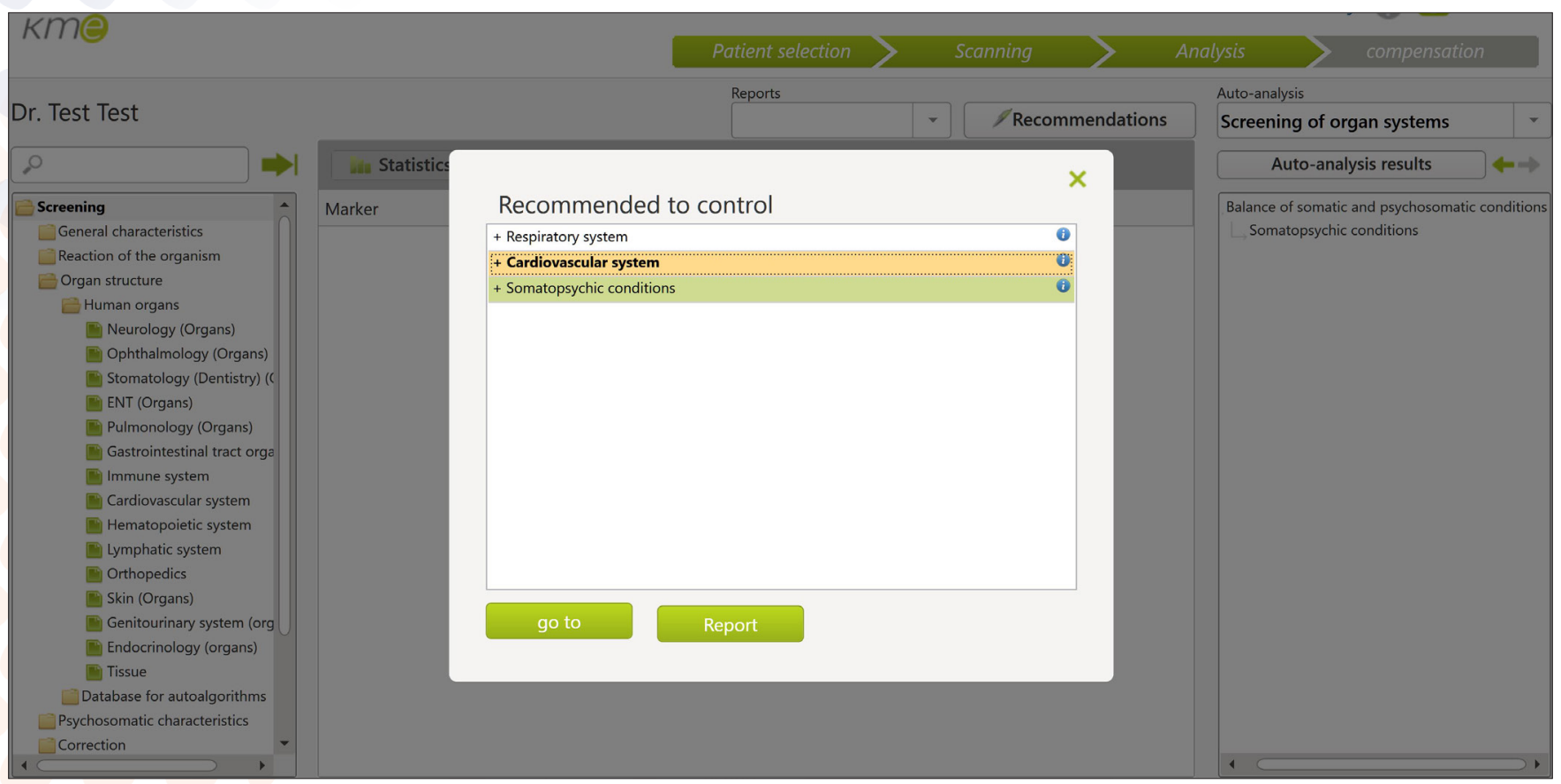

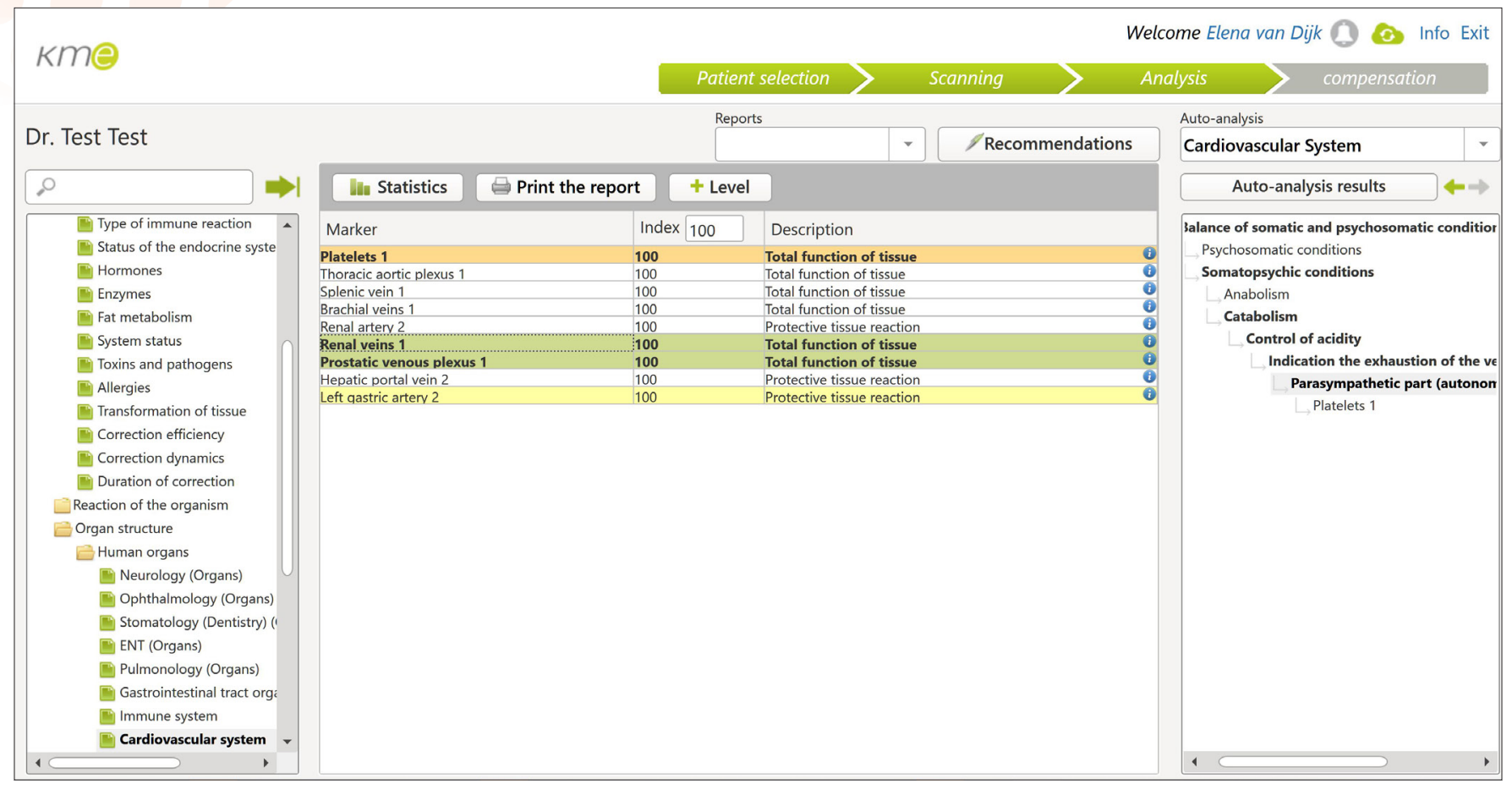

"Dominant" Method and causality chain

The dominant method is a method of analyzing the dynamics and interactions of the processes that contribute to the emergence and development of pathogenic processes and diseases.

This method allows consideration of any given process to become the focus of analysis by taking it into the "dominant" (+level).

Any of the processes in the causal chain, can also be opened in the next "dominant" (+level), and then considered for its own chain of causality. 
The resulting analysis of the process's interaction may determine the causes of the emergence in both actual pathological processes, and the main causes of changes in the general health condition.

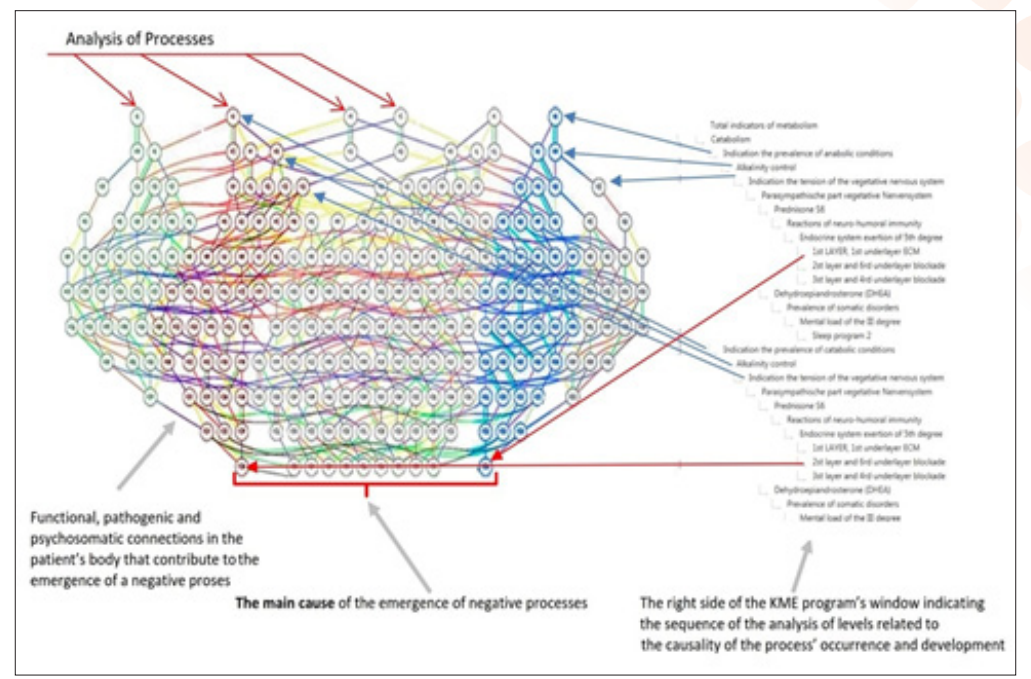

Method of "Compensatory adjustment" (optional experimental addition)

We know that the human body or any biological system (animals, plants and others) is an adaptive, self-regulating system with a multitude of different factors. Pathology is the factor that disturbs the balance in this system (which is causing imbalance). Accordingly, our adaptive system reacts to all these disturbing factors in the pursuit to return the system to the state of balance.

As a result of this reaction, we get the external characteristics of a particular disease, such as fever and pain (as signaling factors in critical changes of the organ's condition), etc.

Effect of the Compensation on a pathology in the body changes the balance between the activity of the pathology and the immune response of the body. This targeting with the help of choosing the "optimal approach" is a compensatory adjustment. A user of the ERI system could achieve the desired results, by helping to eliminate not the symptoms, but rather the causes of their occurrence.

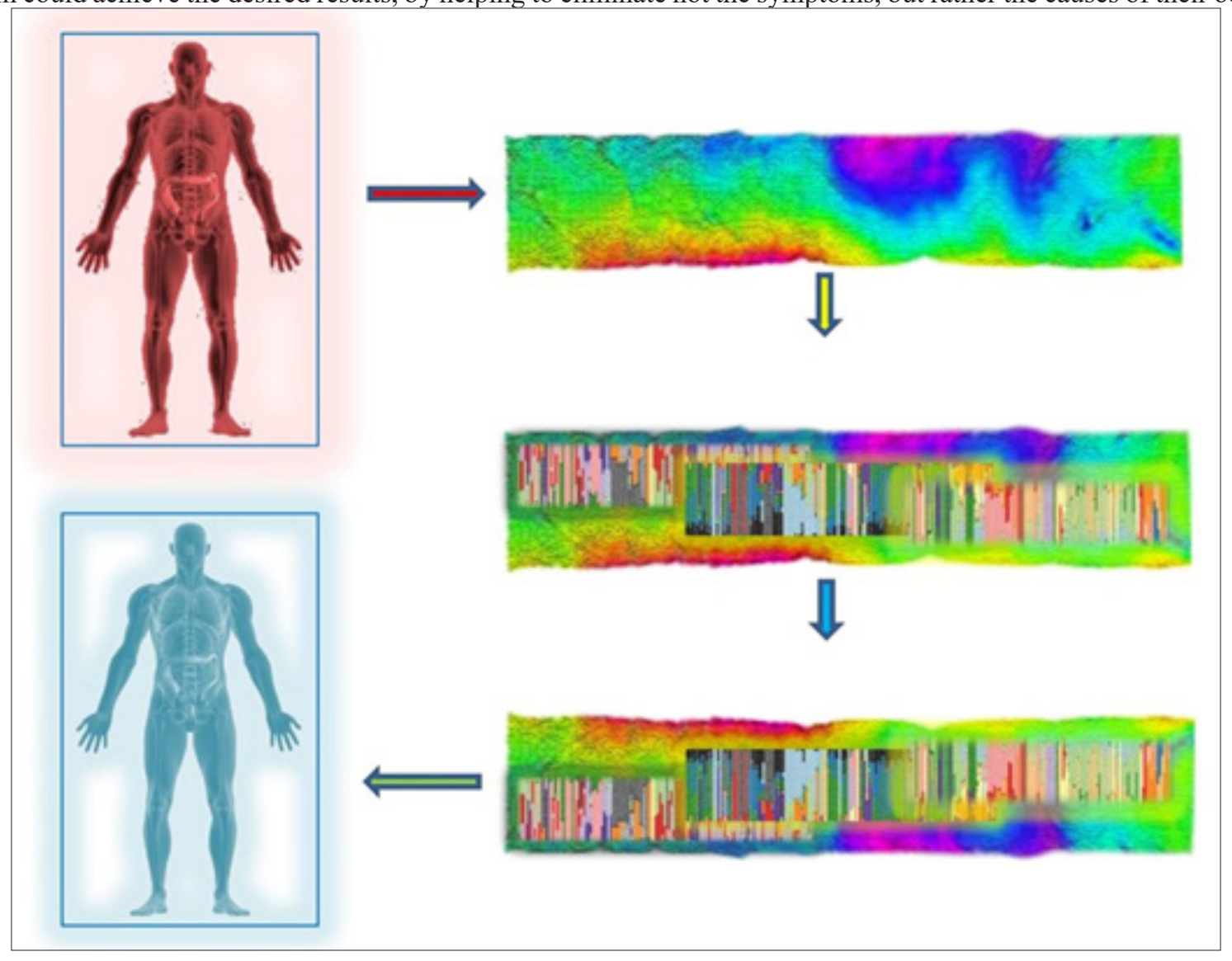


Diagnosis is always in hands of doctor. No system would replace human!

Only a professional health care provider can make decisions about the patient's state of health and establish proper diagnosis, prescribe methods and means of treatments in accordance with the requirements of the acceptable medical standards.

The ERI system is a complementary addition to any procedures. It is presented only as alternative information base system. It does not make diagnose. Only medical specialist can make a decision about the patient's conditions.

Analytical Cloud System ERI - a novel approach to an optimization of health \& quality of life.

Copyright: (2021 Elena van Dijk. This is an open-access article distributed under the terms of the Creative Commons Attribution License, which permits unrestricted use, distribution, and reproduction in anymedium, provided the original author and source are credited. 